\title{
Spontaneous Skyrmion Ground States in Magnetic Metals
}

\author{
U.K. Rößler and A.N. Bogdanov* \\ IFW Dresden, P.O. Box 270116, D-01171 Dresden, Germany \\ C. Pfleiderer \\ Physik Department E21, Technische Universität München, \\ James-Franck-Strasse, D-85748 Garching, Germany
}

(Dated: February 4, 2008)

${ }^{*}$ Permanent address: Donetsk Institute for Physics and Technology, 340114 Donetsk, Ukraine 
Since the 1950s Heisenberg and others have attempted to explain the appearance of countable particles in quantum field theory in terms of stable localized field configurations [1]. As an exception Skyrme's model succeeded to describe nuclear particles as localized states, so-called 'skyrmions', within a non-linear field theory [2]. Skyrmions are a characteristic of non-linear continuum models ranging from microscopic to cosmological scales $[3,4,5,6]$. Skyrmionic states have been found under non-equilibrium conditions, or when stabilised by external fields or the proliferation of topological defects. Examples are Turing patterns in classical liquids [7], spin textures in quantum Hall magnets [8], or the blue phases in liquid crystals [9], respectively. However, it is believed that skyrmions cannot form spontaneous ground states like ferromagnetic or antiferromagnetic order in magnetic materials. Here, we show theoretically that this assumption is wrong and that skyrmion textures may form spontaneously in condensed matter systems with chiral interactions without the assistance of external fields or the proliferation of defects. We show this within a phenomenological continuum model, that is based on a few material-specific parameters that may be determined from experiment. As a new condition not considered before, we allow for softened amplitude variations of the magnetisation - a key property of, for instance, metallic magnets. Our model implies that spontaneous skyrmion lattice ground states may exist quite generally in a large number of materials, notably at surfaces and in thin films as well as in bulk compounds, where a lack of space inversion symmetry leads to chiral interactions.

The possibility that particle-like states may form spontaneously in continuous fields has motivated intense theoretical efforts in the past. Derrick and Hobart established by rather general arguments that particle-like configurations are not stable in the majority of nonlinear field models [10, 11]. However, a few exceptions have been found. Skyrme showed that particle-like excitations of continuous fields exist in the presence of certain non-linear interactions 2]. As a drawback, the interactions considered by Skyrme are physically not transparent, because they involve higher order derivative terms that are technically intractable. Therefore, Skyrme's approach is not viable in the context of ordered states in condensed matter that are ruled by short range interactions. In contrast, a physically transparent exception to the Derrick-Hobart theorem has been recognized in systems with bro- 
ken inversion symmetry, where chiral interactions lead to skyrmion excitations in condensed matter systems [12, 14, 16]. Chiral interactions exist in many different systems, e.g., (i) spin-orbit interactions in non-centrosymmetric materials, also referred to as DzyaloshinskyMoriya (DM) interactions [13], (ii) in non-centrosymmetric ferroelectrics, (iii) for certain structural phase transitions, (iv) in chiral liquid crystals, and (v) in the form of ChernSimons terms in gauge field theories (see section $\mathrm{V}$ in [20] for references). Yet, it was concluded that spontaneous skyrmion ground states are not stable, but can only be induced by an appropriate applied field [14] or the proliferation of topological defects [9]. Finally, a somewhat different mechanism has been found in quantum Hall ferromagnets. Here charge as an additional degree of freedom allows to stabilises skyrmions in an external field. As for the other examples skyrmions in quantum Hall ferromagnets do not form spontaneous ground states.

We have chosen to study skyrmions that are driven by chiral interactions, because the magnetism of condensed matter systems provides perhaps the richest setting of physics problems in which different kinds of chiral interactions may be studied. Present day experimental and theoretical studies [9, 14] have led to the view that only one-dimensional (uniform) modulations exist as spontaneous ground states in these systems (for an illustration see Fig. 1a). In the following, we show that previous studies have been incomplete and that skyrmions may form spontaneously in magnets with chiral interactions in terms of multi-dimensional modulations, when the amplitude of the magnetisation is exceptionally soft. The mathematical structure and the physical nature of the skyrmion texture constitute the first example for a spontaneous skyrmion ground state in an ordered condensed matter system. The example we identify is in particular important, because it can be tested experimentally in comparatively simple laboratory experiments.

In our calculation we consider systems with a hierachy of well-separated energy and length scales, where ferromagnetic exchange favouring spin alignment is the strongest scale, followed by chiral interactions favouring spin rotations on intermediate scales, and magnetic anisotropies and dipolar interactions that determine favourable directions of spins as the weakest scale. Our model treats the magnetic states in chiral magnets within a phenomenological quasi-classical continuum approximation. This micromagnetic approach is appropriate for chiral magnets where the helical modulations are large as compared to atomic distances, i.e., it applies to modulations with typical lengths of a few hundred up to 
several thousand $\AA$ observed in many chiral helix magnets [13, 15, 16]. The model, hence, specifically addresses intermediate length scales.

We write the magnetic free energy density in the form

$$
f=A m^{2} \sum_{i, j}\left(\partial_{i} n_{j}\right)^{2}+\eta A(\nabla m)^{2}+f_{D}(\mathbf{m})+f_{0}(m)
$$

where the first and second term in $\eta$ and $A(\eta, A>0)$, respectively, describe the magnetic stiffness with $\mathbf{n}$ being a unit vector along the magnetization, $\mathbf{m}=m \mathbf{n})$. The third term $f_{D}(\mathbf{m}) \equiv D \mathcal{L}(\mathbf{m})$ in the free energy describes the chiral interactions, where the Dzyaloshinsky-Moriya constant $D$ determines its handedness and strength. The DMinteractions can be expressed in terms of so-called Lifshitz invariants, i.e., linear, antisymmetric gradient terms of the magnetization $\mathcal{L}_{i j}^{(k)}=\left(m_{i} \partial_{k} m_{j}-m_{j} \partial_{k} m_{i}\right)$ where $i, j, k$ are combinations of Cartesian coordinates $x, y, z$ that are consistent with the symmetry class of the system under consideration. The fourth term $f_{0}(m)$, finally, includes nongradient terms of the free energy and may be expanded according to Landau theory in even powers of $m$. We neglect weaker contributions such as magnetic anisotropies, or dipolar interactions and address their importance in the discussion of our results.

The magnetic stiffness terms in $\eta$ and $A$ are motivated as follows. It is convenient to consider at first local moments, characterised by a fixed magnetisation modulus. For isotropic local moment ferromagnets (LMFM) in the ordered state it is well-known [17], that the magnetisation as described in a continuum limit of the Heisenberg model displays changes of orientation and modulus due to magnon-magnon scattering. This situation is described by the gradient energy given above for $\eta=1$. Patashinskii and Pokrovskii [18] further showed that magnon-magnon scattering in LMFM also forces the longitudinal stiffness to vanish in the limit of infinite wavelength, i.e., $\eta=1$ is valid in this limit also (see also, Zwerger [19]). However, the SU(2) symmetry of the spin system enforces that the longitudinal stiffness is the result of higher order transverse processes. Further mechanisms on top of magnon-magnon scattering, such as coupling to the particle-hole continuum in itinerant-electron systems, dipolar interactions and defects, therefore additionally reduce the longitudinal stiffness before it vanishes altogether in the limit of infinite wavelength. A minimal description of this additional longitudinal softening requires the second gradient term written in $\eta$ with $\eta<1$, where $\eta$ expresses the ratio between longitudinal and transverse stiffness of the ordered magnetic state. For an extensive discussion of the technical 
validity of the two gradient terms used here and their relationship to conventional spin fluctuation theories of itinerant-electron magnets, we refer to the supplementary information of this paper [20].

We have also analysed 20] published data of the longitudinal susceptibility in the localmoment ferromagnet EuS 21] and the itinerant-electron ferromagnets $\mathrm{Ni}$ [22] and $\mathrm{MnSi}$ [23]. Our analysis establishes values of $\eta$ for EuS, Ni and MnSi of 0.925, 0.65 and 0.4, respectively, i.e, important reductions of the longitudinal stiffness exist in all real materials. The experimental data for these archetypical ferromagnets show that the regime $\eta<1$ in our model is valid and relevant for many different types of magnetic systems. In particular, the very prominent longitudinal softening in itinerant-electron magnets $\mathrm{Ni}$ and $\mathrm{MnSi}$ has not yet been appreciated before. In the presence of chiral interactions the reductions of longitudinal stiffness become of tantamount importance, because they facilitate the formation of spontaneous skyrmion ground states.

For our model we first show that certain individual skyrmions represent localised regions of reduced ground state energy on a background of vanishing magnetization. General consideration of the stability of particle-like solutions as function of dimension against compression/stretching [12] strongly suggest that two-dimensional solutions are the most stable. We therefore begin by considering an individual skyrmion of cylindrical symmetry, as shown in Fig. $1 \mathrm{~b}$ and $\mathrm{c}$ to explain the basic physical ideas. As explained in further detail below, the stability of such a cylindrical structure establishes the possibility of spontaneous skyrmion lattices in all noncentrosymmetric crystallographic classes that allow chiral interactions [16].

Expanding the contribution $f_{0}(m)$ according to Landau theory for small $m$ as $f_{0}=a(T-$ $\left.T_{C}\right) m^{2}+b m^{4}+\ldots$ leads in the absence of chiral interactions $\left(f_{D}=0\right)$ to the conventional Curie temperature $T_{C}$ of centrosymmetric systems. Thus, when the temperature drops below $T_{C}$ the energy density of a ferromagnetically spin-aligned state is lowest. Chiral interactions $\left(f_{D}<0\right)$ favouring rotations of the moments with respect to each other reduce the energy density further. This stabilization of twisted magnetic states occurs only by the competition of the DM-interactions with the exchange, expressed by the form of the gradient energy. Therefore, the exact form of $f_{0}(m)$ is not decisive for the stability of skyrmions. In particular a form of $f_{0}(m)$ including higher order terms with $b<0$ and a stabilizing term $m^{6}$ for a first-order transition will change only quantitative relations and the details of the phase-diagram. Possibly, it may lead to the discontinuous nucleation of skyrmions. Figure 1 
illustrates the twisted magnetic structures with rotating magnetization and the associated energy densities. For rotations along a single given direction, shown in Fig. 1 a, the reduction in energy density, shown in Fig $1 \mathrm{~d}$, is uniform. The transition to the ferromagnetic state is consequently pre-empted by a transition to a one-dimensionally modulated state at a temperature

$$
T_{h}=T_{C}+\frac{D^{2}}{4 A a}
$$

As shown in Fig. $1 \mathrm{~b}$ and $\mathrm{d}$ rotations of the magnetisation in two dimensions reduce the energy density even further near its nucleation point at the center of the cylindrical structure $\rho=0$. Considering only the energy reduction at $\rho=0$ we obtain as an upper limit for the temperature, $T_{D}$, for the onset of this so-called double-twisting

$$
T_{D}=T_{C}+\frac{D^{2}}{2 A a}
$$

The characteristic temperatures $T_{h}$ and $T_{D}$ depend only on a few physically meaningful, material specific parameters that may be determined from experiment. The ratio $A / D$ measures the pitch of the chiral modulations in the ordered helical state, while $D / a$ measures the chirality of magnetic fluctuations in the paramagnetic state. Hence, $D / a$ is related to a chiral component of the susceptibility. The parameters $a$ and $b$ are the initial susceptibility and mode coupling parameter, respectively. This permits estimates of $T_{h}$ and $T_{D}$ from experiments. An example is given below.

As shown in Fig. $1 \mathrm{~d}$ the energy reduction due to a double-twist is no longer uniform. In fact, for fixed magnetisation amplitude the energy reduction turns into an excess of the energy density at larger distances $\rho$ from the center, which outweighs the initial reduction. This destabilises the double-twist structure altogether, which is the reason why magnetic skyrmions prior to the work reported here were believed to be unstable. Skyrmion textures may only form spontaneously when no additional energy is required for double-twist rotations at large $\rho$. The latter may be achieved by permitting the magnitude of the moment to be soft. As shown in Fig. $1 \mathrm{c}$ and $1 \mathrm{~d}$ skyrmions under these conditions have the lowest energy density, when the moment decreases with increasing distance from the centre.

The results of a comprehensive numerical analysis of the free energy for skyrmions taking into account the variation of the energy density with $\rho$ for differing $\eta$ are summarised in Fig. 2 and 3 (see Eqn. (11) and Methods section). This analysis establishes rigorously that stable cylindrical skyrmions form spontaneously in a finite temperature interval $T_{t}<T<T_{S}$ of the 
phase diagram Fig. 2, where the limits $T_{h}$ and $T_{D}$ are also marked. The magnetic structure of the skyrmions is characterised by a magnetisation vector $\mathbf{m}=(m, \theta, \psi)$ that rotates outward from the axis in all directions, while the modulus $m(\rho)$ shrinks and decreases towards the boundary of the tube at radius $R$ (Fig. 1c). The amplitude of the local magnetisation $m(\rho)$ vanishes continuously when approaching $T_{S}$ from below, as shown in Fig. 3. Thus, the skyrmion nucleation and the formation of the skyrmion texture coincide. The increase of the magnetization with decreasing temperature drives the transition from the skyrmion phase to the uniform helix phase at a lower temperature $T_{t}$. The transition into a onedimensionally modulated state by transforming the structure Fig. $1 \mathrm{~b}$ or c into the helix Fig 1 a cannot be performed by a smooth deformation of the skyrmions, because these two textures have different topology. For this reason, a first-order transition is generally expected at a temperature $T_{t}$. In the phase diagram, the transition line $T_{t}$ has been determined from the approximate condition that the average energy of the circular skyrmion cell is equal to that of the helix. The skyrmion phase vanishes as $T_{S} \rightarrow T_{t}$ in the limit $\eta \rightarrow 1$. This feature of the phase diagram (Fig. 2) concurs with an analytical result by Wright and Mermin for magnets with the properties of fixed moment classical Heisenberg models (Eqn. (11) with $\eta=1$ ) 9]. The result was believed to prove the absence of skyrmion ground states in chiral ferromagnets. However, it applies only to a particular form of the model where $\eta=1$, while the regime $\eta<1$ had not been considered systematically.

Having established that cylindrical skyrmions with soft amplitude can be localised regions of reduced energy we next address the proliferation of these skyrmions to form textures. In a chiral magnet, the formation of an extended skyrmion condensate is subject to: (i) weak residual magnetic anisotropies and dipolar interactions in the crystalline environment, and (ii) the magnetisation in the regime joining the skyrmion tubes. Under the most favourable conditions, the skyrmion condensate may form a regular lattice in analogy to the vortex lattice of type 2 superconductors. In fact, there exists a fundamental analogy between magnetic skyrmion lattices and Abrikosov lattices. The formation of stable spatially modulated states in superconductors and in magnets can be justified by the concept of negative domain wall energies [16, 36, 37]. We illustrate the stability of a skyrmion condensate for this case by the full solution for an extended state in an isotropic system in two dimensions. We note that the solution as shown in Fig. 4 applies also to three-dimensional systems, when the solutions are artificially constrained to remain homogeneous in the third spatial direc- 
tion (perpendicular to the plane shown in Fig. 4). The structure of this solution shows in particular that the core region of the skyrmions is unaffected, as stated above, while the magnetization between the skyrmions mediating their interactions does not even need to be strictly zero for the skyrmion lattice to become the ground state.

The spontaneous formation of skyrmions based on cylinder-symmetric solutions applies to all symmetry classes including higher symmetries, say the cubic and the isotropic case without inversion center, because the core structure of the skyrmions is determined by the two largest energy scales, namely the exchange energy and chiral interactions, while the texture is determined by weaker interactions. Technically the general validity may be seen by noting that the Euler equations for the modulus of the magnetisation $m$ and the polar angle $\theta$ which control the energetic stability of the skyrmions, given by Eqn. (7) and (8) in the Methods section, respectively, are identical for all relevant symmetry classes (see Refs. [12, 16] for a discussion). In contrast, the behaviour of the azimuthal angle $\psi$, which differs for the different symmetry classes, only affects the manner of rotation of the magnetic moments in the radial direction. General considerations of the stability of localized textures for chiral magnets suggest that droplet-like skyrmions are not stable in three dimensions 12]. Therefore, we believe that the two-dimensionally localized skyrmion tubes discussed so far will form extended ground-state condensates in three-dimensional systems. However, in a three-dimensional environment bending and terminating skyrmion tubes imposes a cost in energy that determines the nature of the condensate. The additional cost in energies may be readily reduced by closing the tubes amongst themselves or ending them at surface and defects. In isotropic three-dimensional materials the skyrmion condensate will therefore have a liquid-like or amorphous appearance driven by the frustration between preferred orientations and their elastic interactions in the condensed structure.

Experimental systems where skyrmion ground states may exist will have to display softened longitudinal stiffness together with a hirachy of energy scales in which spin-aligning exchange interactions are dominant followed by subleading chiral interactions causing spin rotations. Further, magnetic anisotropies must be low because they prevent the continuous rotation of the magnetization and, thus, multiply-modulated textures [14, 16, 39]. The elementary mechanism, shown here for a ferromagnet and illustrated in Fig. 1, then applies to any chiral system with a tendency to form twisted structures. In particular, our model also applies to antiferromagnetic crystals with chiral symmetry by replacing the magnetiza- 
tion with a staggered antiferromagnetic vector order parameter 38]. Owing to the broken inversion symmetry at the surfaces of magnetic materials [28, 29] thin films and interfaces are the largest class of condensed matter systems where skyrmion condensates may form spontaneously. Appropriate metallic films of this kind have not been investigated in detail in the regime of interest. A less frequent, but equally promising class of materials are bulk materials with chiral interactions due to a lack of inversion symmetry.

The most powerful technique to test our prediction experimentally is polarised magnetic neutron scattering. For a periodic skyrmion lattice neutron scattering will show long-range order akin to the vortex lattice in superconductors, while the scattering intensity of an amorphous appearance will be akin to partial order in liquid crystals [40]. In the bulk properties subtle evidence for the predicted intermediate phase is expected, when going from paramagnetism to helical magnetic order. Real-space imaging of magnetic textures by magnetic force microscopy, spin sensitive tunneling, magnetic x-ray microscopy, and Lorentz transmission electron microscopy are finally expected to provide evidence of skyrmion lattices akin vortex lattices in the Shubnikov phase of superconductors. The helical structure in the chiral magnet (Fe,Co)Si were recently imaged directly for the first time [42] because of the exceptionally long helix period in this material, but the technique may introduce uncontrolled strains that interfere with the intrinsic properties. It is to be expected that the resolution of improved imaging techniques will soon be sufficient to take pictures of the skyrmions predicted here.

Our study was inspired by recent high pressure experiments in the cubic itinerant-electron helimagnet MnSi. The low temperature resistivity in MnSi changes abruptly from a $T^{2}$-Fermi liquid temperature dependence to a $T^{3 / 2}$ dependence above a pressure $p_{c}=14.6 \mathrm{kbar}$ [32, 33]. The resistivity is unchanged up to at least $3 p_{c}$, suggesting the formation of a stable phase. However, as emphasized in Ref. [32] a $T^{3 / 2}$ resistivity is normally characteristic of spin glasses and amorphous ferromagnets where the conduction electrons undergo a diffusive motion, but not for the ultra-pure MnSi single-crystals investigated, which by all accounts should show the behaviour of a Fermi liquid. The spontaneous formation of an amorphous magnetic state in ultrapure samples would provide a simple explanation that resolves this contradiction of high purity and diffusive charge carrier motion in MnSi. It also explains the stability of the resistivity as a new phase. Magnetic neutron scattering in MnSi further revealed, that large ordered moments are present in the nominally paramagnetic phase. The ordered moments 
are organised such that they lead to scattering intensity on the surface of a sphere with unexplained broad maxima for $\langle 110\rangle$. If we suppose that the easy magnetic axis, $\langle 111\rangle$, does not change as function of pressure, while the magnetism changes from a helical modulation along $\langle 111\rangle$ to cylindrical skyrmion tubes with their axes preferentially along $\langle 111\rangle$ we expect intensity in the great circles perpendicular to $\langle 111\rangle$. These great circles interset along the $\langle 110\rangle$ directions, thus explaining the broad maxima in this location. Finally, as shown above we predict a spontaneous skyrmion phase prior to the onset of helical order. From the values of $T_{C}=29.5 \mathrm{~K}$, the wavelength of the helix $q_{0}=0.039 \AA^{-1}$ and the effective Curie-Weiss moment $p_{\text {eff }}=2.2 \mu_{B}$ we estimate $T_{D}-T_{C} \approx 0.9 \mathrm{~K}[20]$. A hump in the specific heat above $T_{C}$ and and an associated sphere in neutron scattering intensity as observed in ambient pressure experiments in MnSi are consistent with such a phase over a temperature interval of $\sim 1 \mathrm{~K}$ [20, 31|. We note that recent theories on the partial magnetic order in MnSi by Tewari et al. [26] and Binz et al.[27] also suggest inhomogeneous twisted states with modulated amplitude. These theories rely on unsubstantiated higher-order interaction terms and, therefore, share the problems with the corresponding non-linear field theories without identifying the basic mechanism of stable skyrmions in such textures. The evidence for a skyrmion ground state in MnSi discussed here resolves all of the existing contradictions, but a definitive proof will require a host of novel techniques listed above.

We finally note, that the argument for the existence of skyrmion textures in ferromagnetic metals is closely related to the blue phases of chiral nematic crystals [9, 35]. The standard continuum model of chiral nematics admits localized structures as vortex tubes [35], where the energy disadvantage by double-twisting a configuration has been recognised early on [9]. However, in the blue phases topological defects in the form of disclination lines, which do not exist in magnetic systems, allow to overcome the disadvantage in energy and stabilise extended textures. In chiral magnets skyrmion textures appear instead as condensate of strictly localized skyrmions with lines or sheets of vanishing magnetic order between them (Figure 4). Fluctuations and the competition betwen different frustrated couplings entail the possibility of internal transitions between various textures with liquid, glassy, or latticelike organization of skyrmions. Thus, the predicted magnetic skyrmion textures promise rich phase diagrams, similar to those of liquid crystals and Abrikosov lattices of flux-lines in type-II superconductors [36]. 


\section{Acknowledgements}

We wish to thank H. Eschrig, I. Fischer, A. Möbius, A. Rosch, H. v. Löhneysen, M. Vojta, and W. Zwerger for support and discussions. We are particularly greatful to P. Böni

for discussions on his studies of longitudinal magnetic fluctuations in EuS, Ni and MnSi . AB thanks the DFG-Graduiertenkolleg GRK 284 'Kollektive Pänomene im Festkörper' for financial support. CP acknowledges support in the framework of a Helmholtz-HochschulNachwuchsgruppe at the Universität Karlsruhe in the initial part of this project. 


\section{Methods}

Cubic noncentrosymmetric magnets (e.g., MnSi and other B20 compounds [15])) belong to the crystallographic class $T$. For this class the Dzyaloshinsky-Moriya energy is

$$
f_{D}=D\left(\mathcal{L}_{y x}^{(z)}+\mathcal{L}_{x z}^{(y)}+\mathcal{L}_{z y}^{(x)}\right) \equiv D \mathbf{m} \cdot \operatorname{rot} \mathbf{m}
$$

where $D$ is the Dzyaloshinsky constant [15]. The helix solutions for these systems are described by a linear wave with a wave number $q_{0}=D /(2 A)$ and constant magnetisation amplitude $m^{2}=2 a\left(T_{h}-T\right) / b[9,15]$. For all uniaxial systems the invariants $f_{D}$ are listed in [16]. In particular, for uniaxal magnets with $C_{n v}$ symmetry $(n=3,4,6)$ one has $f_{D}=$ $D\left(\mathcal{L}_{x z}^{(x)}-\mathcal{L}_{y z}^{(y)}\right)$. The $n$-fold axis is directed along $z$. Examples are $\mathrm{Tb}_{3} \mathrm{Al}_{2}$ or $\mathrm{Dy}_{3} \mathrm{Al}_{2}$ with tetragonal noncentrosymmetric structure $\mathrm{C}_{4 v}^{4}$, 14] or the superconducting antiferromagnet $\mathrm{CePt}_{3} \mathrm{Si}$, space group P4mm [1]]. The structure of the cylindrically symmetric skyrmions is given by solutions of the Euler equations for the free energy Eqn. (11). The magnetization vector $\mathbf{m}=(m, \theta, \psi)$ is aligned along the cylinder or $z$-axis in the skyrmion center, $\rho=0$. In cubic and uniaxial systems solutions for a skyrmion homogeneous along the $z$-direction are given by $m(\rho), \theta(\rho), \psi=\operatorname{sign} \phi+\phi_{0}$, where sign $=+$ or - and $\phi_{0}$ is a constant, both determined by the specific symmetry class 14,16$]$. The dependence $m(\rho)$ and $\theta(\rho)$ describes the variation of the amplitude of the magnetic moments and their angle as function of the distance $\rho$ to the cylinder axis in cylindrical coordinates, respectively. After substitution of the solutions for $\psi(\phi)$ and an integration with respect to $z$ and $\phi$ the energy of the skyrmion is reduced to the following functional $\mathcal{F}_{s}=2 \pi L \int_{0}^{\infty} f_{s}(m, \theta) \rho d \rho$ with energy density

$$
\begin{array}{r}
f_{s}=\eta A\left(\frac{d m}{d \rho}\right)^{2}+A \mathcal{G}\left(\theta, \theta_{\rho}\right) m^{2}+q_{0}^{-2} f_{0}(m), \\
\mathcal{G}\left(\theta, \theta_{\rho}\right)=\left(\frac{d \theta}{d \rho}\right)^{2}+\frac{\sin ^{2} \theta}{\rho^{2}}-2\left[\left(\frac{d \theta}{d \rho}\right)+\frac{\sin \theta \cos \theta}{\rho}\right],
\end{array}
$$

where $L$ is the length of the skyrmion along $z$-direction, and the spatial coordinate $\rho$ is measured in $q_{0}^{-1}$ units. The Euler equations for the functional (6)

$$
\begin{gathered}
\eta\left[\left(\frac{d^{2} m}{d \rho^{2}}\right)+\frac{1}{\rho}\left(\frac{d m}{d \rho}\right)\right]-A \mathcal{G}\left(\theta, \theta_{\rho}\right) m-q_{0}^{-2} \frac{\partial f_{0}}{\partial m}=0 \\
m\left[\left(\frac{d^{2} \theta}{d \rho^{2}}\right)+\frac{1}{\rho}\left(\frac{d \theta}{d \rho}\right)-\frac{\sin \theta \cos \theta}{\rho^{2}}-\frac{2 \sin ^{2} \theta}{\rho}\right]+2\left[\left(\frac{d \theta}{d \rho}\right)-1\right]\left(\frac{d m}{d \rho}\right)=0
\end{gathered}
$$


with the boundary conditions $\theta(0)=0, m(0)=m_{0}, m(R)=0$ describe the structure of the isolated skyrmion and yield the energy as a function of $m_{0}$ and $R$. The equilibrium skyrmion profiles are derived by minimization of $\mathcal{F}_{s}\left(m_{0}\right)$ with respect to $m_{0}$ and $R$; the radially integrated energy densities of the vortices per lengths (Figure $1 \mathrm{~d}$ ) are given by $w(\rho)=\left(2 / \rho^{2}\right) \int_{0}^{\rho} f_{s}(m, \theta) \rho d \rho$. Full solutions for extended two-dimensional models have been constructed numerically using a finite-difference scheme on rectangular lattices with dynamically optimized lattice parameters and periodic boundary conditions. The search for ground-states and checks on their stability were done using a continuous variable Monte Carlo simulated annealing method and mesh-refinement.

The unit length used throughout this paper is $1 / q_{0}$, which is the inverse of the twisting length in chiral magnets with Dzyaloshinsky-Moriya couplings. Energy and temperature are given in equivalent units of $D^{2} /(2 A a)$ as in Eqs. (2) and (3). 


\section{References}

[1] W. Heisenberg, Einführung in die einheitliche Feldtheorie der Elementarteilchen. (Hirzel, Stuttgart 1967).

[2] T. H. Skyrme, A Non-linear field theory. Proc. Royal Soc. London, Ser. A 260, 127-138 (1961).

[3] R. Durrer, M. Kunz, A. Melchiorri, Cosmic structure formation with topological defects. Phys. Rep. 364, 1-81 (2002).

[4] C. Bäuerle, Yu. M. Bunkov, S. N. Fisher, H. Godfrin, G. R. Pickett, Laboratory simulation of cosmic string formation in the early Universe using superfluid ${ }^{3}$ He. Nature $\mathbf{3 8 2}, 332-334$ (1996).

[5] L. Brey, H. A. Fertig, R. Cote, and A. H. MacDonald, Skyrme Crystal in a Two-Dimensional Electron Gas. Phys. Rev. Lett. 75, 2562-2565 (1995).

[6] U. Al'Khawaja and H. T. C. Stoof, Skyrmions in a Ferromagnetic Bose-Einstein Condensate. Nature 411, 918-920 (2001).

[7] M. C. Cross, P. C. Hohenberg, Pattern formation outside of equilibrium. Rev. Mod. Phys. 65, 852-1112 (1993).

[8] S. L. Sondhi, A. Karlhede, S. A. Kivelson, and E. H. Rezayi, Skyrmions and the crossover from the integer to the fractional quantum Hall effect at small Zeeman energies. Phys. Rev. B 47, 16419-19426 (1993).

[9] D. C. Wright, N. D. Mermin, Crystalline liquids — the blue phases. Rev. Mod. Phys. 61, 385-432 (1989).

[10] R.H. Hobart, On instability of a class of unitary field models. Proc. Phys. Soc. London 82, 201-202 (1963).

[11] G.H. Derrick, Comments on nonlinear wave equations as models for elementary particles. $J$. Math. Phys. 5, 1252-1253 (1964).

[12] A. Bogdanov, New localized solutions of the nonlinear field-equations. JETP Lett. 62, 247251 (1995).

[13] I. E. Dzyaloshinskii, Theory of helicoidal structures in antiferromagnets. Sov. Phys. JETP 19, 960-971 (1964) 
[14] A. Bogdanov and A. Hubert, Thermodynamically stable magnetic vortex states in magnetic crystals. J. Magn. Magn. Mater. 138, 255-269 (1994).

[15] P. Bak, M. H. Jensen, Theory of helical magnetic-structures and phase-transitions in MnSi and FeGe. J. Phys. C: Solid St. Phys., 13, L881-885 (1980).

[16] A. N. Bogdanov and D. A. Yablonskii, Thermodynamically stable "vortices" in magnetically ordered crystals. The mixed state of magnets. Sov. Phys. JETP 68, 101-103 (1989).

[17] A. I. Akhiezer, V. G. Bar'yakhtar, S. V. Peletminskii, Spin Waves (North Holland Publ., Amsterdam 1968).

[18] A. Z. Patashinskii, V. L. Pokrovskii, Longitudinal susceptibility and correlations in degenerate systems. Sov. Phys. JETP 37, 733-736 (1974). The phenomenon was independently discovered for Goldstone particles in high energy physics where it gives rise to logarithmic singularities because one is in four dimensions. See L. F. Li and H. Pagels, Perturbation Theory about a Goldstone Symmetry. Phys. Rev. Lett. 26, 1204-1208 (1971).

[19] W. Zwerger, Anomalous Fluctuations in Phases with a Broken Continuous Symmetry. Phys. Rev. Lett. 92, 027203/1-4 (2004).

[20] U. K. Rößler, A. N. Bogdanov, C. Pfleiderer, Supplementary Information to: Spontaneous Skyrmion Ground States in Magnetic Metals. http://xxx.lanl.gov/abs/cond-mat/0603104 (2006).

[21] P. Böni, B. Roessli, D. Görlitz, and J. Kötzler. Damping of spin waves and singularity of the longitudinal modes in the dipolar critical regime of the Heisenberg ferromagnet EuS, Phys. Rev. B, 65, 144434/1-9 (2002).

[22] P. Böni, J. L. Martinez, J. M. Tranquada, Longitudinal spin fluctuations in Ni. Phys. Rev. B 43, 575-584 (1991).

[23] F. Semadeni, P. Böni, Y. Endoh, B. Roessli, G. Shirane. Direct observation of spin-flip excitations in MnSi. Physica B 267-268, 248-251 (1999).

[24] S. S. Saxena, P. Agarwal, K. Ahilan, F. M. Grosche, R. K. W. Haselwimmer, M. J. Steiner, E. Pugh, I. R. Walker, S. R. Julian, P. Monthoux, G. G. Lonzarich, A. Huxley, I. Sheikin, D. Braithwaite, J. Flouquet, Superconductivity on the border of itinerant-electron ferromagnetism in $\mathrm{UGe}_{2}$. Nature 406, 587-592 (2000).

[25] F. Lévy, I. Sheikin, B. Grenier, A. D. Huxley, Magnetic field-induced Superconductivity in the ferromagnet URhGe. Science, 309, 1343-1346 (2005). 
[26] S. Tewari, D. Belitz, T.R. Kirkpatrick, Blue Quantum Fog: Chiral Condensation in Quantum Helimagnets. Phys. Rev. Lett. 96, 047207/1-4 (2006).

[27] B. Binz, A. Vishwanath, V. Aji, Theory of the helical spin crystal: a candidate for the partially ordered state of MnSi. http://xxx.lanl.gov/abs/cond-mat/0602529 (2006).

[28] A. Fert, Metallic Multilayers. in: A. Chamberod, J. Hillairat (Eds.), Materials Science Forum 59-60, 439 (1990).

[29] A. N. Bogdanov and U. K. Rößler, Chiral symmetry breaking in magnetic thin films and multilayers. Phys. Rev. Lett. 87, 037203/1-4 (2001).

[30] B. Lebech, J. Bernhard, T. Freltoft, Magnetic structures of cubic FeGe studied by small-angle neutron scattering. J. Phys.: Condens. Matter 1, 6105-6122 (1989).

[31] B. Lebech, Magnetic Ordering in Nearly Ferromagnetic Antiferromagnetic Helices, in Recent Advances in Magnetism of Transition Metal Compounds. (Eds. A. Kotani, N. Suzuki) 167-178 (World Scientific, Singapore, 1993).

[32] C. Pfleiderer, S. R. Julian, G. G. Lonzarich, Non-Fermi liquid nature of itinerant-electron ferromagnets. Nature 414, 427-430 (2001).

[33] N. Doiron-Leyraud, et al., Fermi-lqiud breakdown in the paramagnetic phase of a pure metal. Nature 425, 595-599 (2003).

[34] C. Pfleiderer, D. Reznik, L. Pintschovius, H. v. Löhneysen, M. Garst, A. Rosch, Partial order in the non-Fermi-liquid phase of MnSi. Nature 427, 227-231 (2004).

[35] R. M. Hornreich, M. Kugler, S. Shtrikman, Localized instabilities and the order-disorder transition in cholesteric liquid crystals. Phys. Rev. Lett. 48, 1404-1407 (1982).

[36] A. A. Abrikosov, On the magnetic properties of superconductors of the second group. Sov. Phys. JETP 5, 1174-1183 (1957).

[37] A. N. Bogdanov, U. K. Rößler, C. Pfleiderer, Modulated and localized structures in cubic helimagnets. Physica B 359-361, 1162-1164 (2005).

[38] A. N. Bogdanov, U. K. Rößler, M. Wolf, K.-H. Müller, Magnetic structures and reorientation transitions in noncentrosymmetric uniaxial antiferromagnets. Phys. Rev. B 66, 214410/1-16 (2002).

[39] Note that dipolar interactions do not commute with ferromagnetic and antiferromagnetic order. Further, dipolar interactions are larger the larger the ordered magnetic moment. This suggests that $\eta<1$ even in the limit $q \rightarrow 0$. While classical dipolar interactions may suppress 
the formation of certain skyrmions they favour the skyrmions relevant for isotropic and cubic ferromagnets, as shown in Figure 4, which have a structure that closes the magnetic flux (with $\phi= \pm \pi$, see Methods section).

[40] P. M. Chaikin, T. C. Lubensky, Principles of Condensed Matter Physics, Cambridge University Press, (1995).

[41] E. Bauer, G. Hilscher, H. Michor, C. Paul, E. W. Scheidt, A. Gribanov, Y. Seropegin, H. Noel, M. Sigrist, P. Rogl, Heavy fermion superconductivity and magnetic order in noncentrosymmetric CePt3Si. Phys. Rev. Lett. 92, 027003/1-4 (2004).

[42] M. Uchida, Y. Onose, Y. Matsui, Y. Tokura, Real-Space Observation of Helical Spin Order. Science 311, 359-361 (2006). 

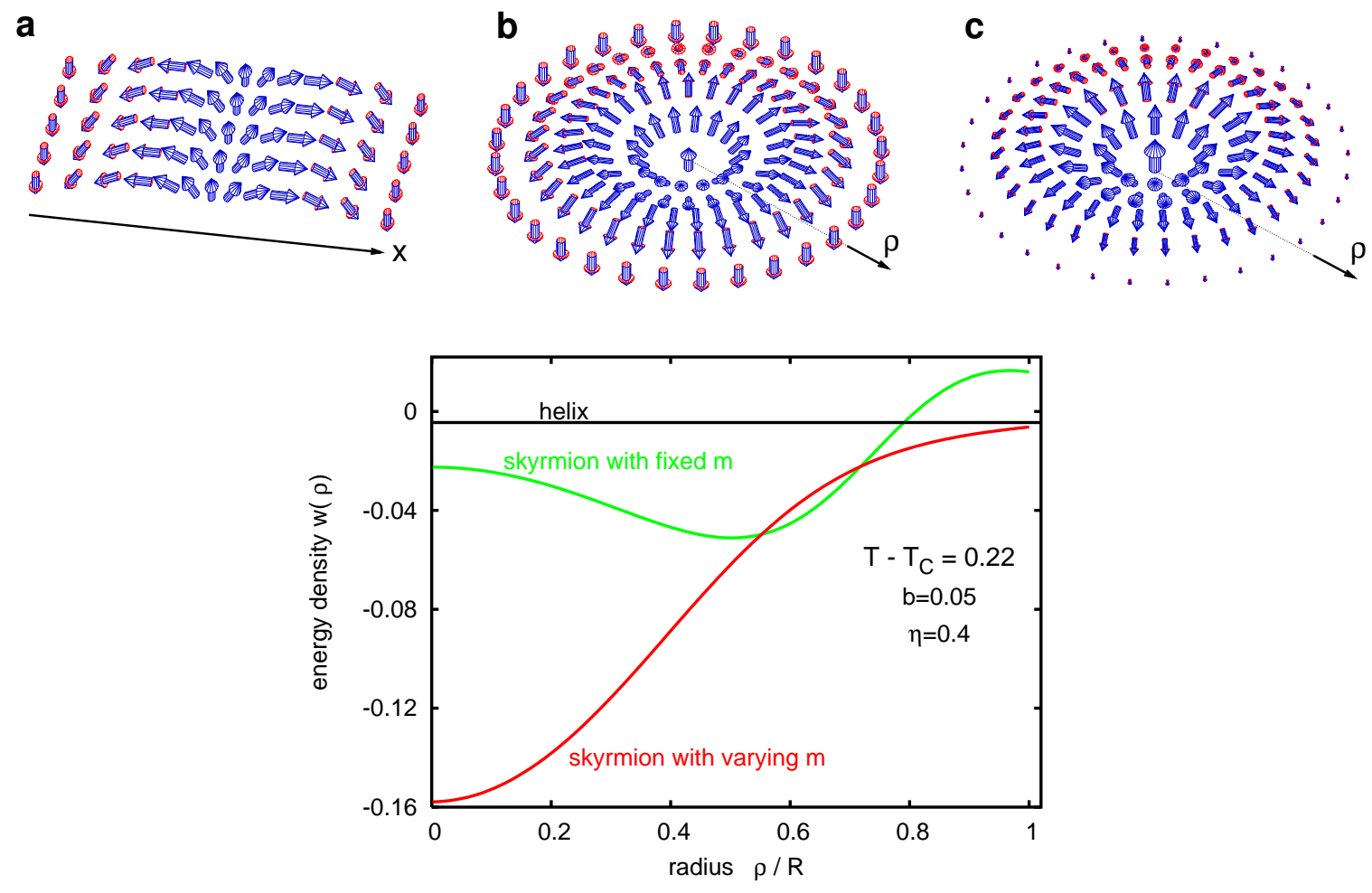

Fig. 1 
FIG. 1: Chiral modulated structures for noncentrosymmetric ferromagnets $(\mathbf{a}-\mathbf{c})$. a, onedimensional modulated structure propagating along a direction $x$ and with a constant modulus of magnetic moments. b, Circular cross section through a skyrmionic excitation with fixed modulus. It can be visualized by rolling one half-period of a helix modulation as in a into a cylindrical tube with propagation axes now pointing radially out in all directions $\rho$ from the center. c, A circular skyrmion cell. The magnetization has maximum modulus at the skyrmion axis and rotates along the radial directions $(\psi=\phi)$. The modulus $m$ gradually decreases with increasing distance from the center, and equals zero at the boundaries of the cell. The chirality (handedness) of the cylindrical skyrmions shown here may be seen as follows: when the fingers on a hand follow the moments rolling outward the thumb defines a winding sense (chirality) around the center (note that space inversion is not satisfied with respect to points in the center as this winding direction would have to be reversed upon space inversion). The magnetic structures $\mathbf{a}, \mathbf{b}, \mathbf{c}$ can exist in noncentrosymmetric crystals of class $\mathrm{C}_{n v}$. The chiral magnets $\mathrm{Tb}_{3} \mathrm{Al}_{2}$ or $\mathrm{Dy}_{3} \mathrm{Al}_{2}$ with tetragonal noncentrosymmetric structure $\mathrm{C}_{4 v}^{4}$ belong to this class [14]. For this crystallographic class the magnetization rotates around an axis, that is perpendicular to the propagation directions in the one-dimensionally modulated state and tangentially for the cylindrical skyrmions. In noncentrosymmetric crystals from the classes $\mathrm{D}_{n}$ and in cubic or isotropic systems, the magnetization in the helix and the skyrmions rotates in the planes perpendicular to the propagation directions, i.e., about the propgagation directions. Such structures can exist in the noncentrosymmetric ferromagnets with B20-structure, in particular in $\mathrm{MnSi}$ [15]. For other crystallographic symmetries more involved structures can occur. All of these magnetic structures in three-dimensional crystals can be described either as one-dimensional modulated state, which is a helix, or as cylindrical skyrmions. Phenomenological theory allows to analyse the stability of these structures for different crystallographic classes within a common mathematical framework, see Methods section and Ref. 16]. d, comparison for the local energy density per unit volume for the helical modulation, a, and fixed and varying magnetic moment $m, \mathbf{b}$ and $\mathbf{c}$, respectively, in circular skyrmion cells (radius $R$ ) along a radial direction $\rho$. The corresponding helical state has a constant energy density which is always higher than the energy density in the center of the skyrmions. For fixed-moment skyrmions this energy gain is offset by a large excess energy near the boundary of the cell at $R$. 


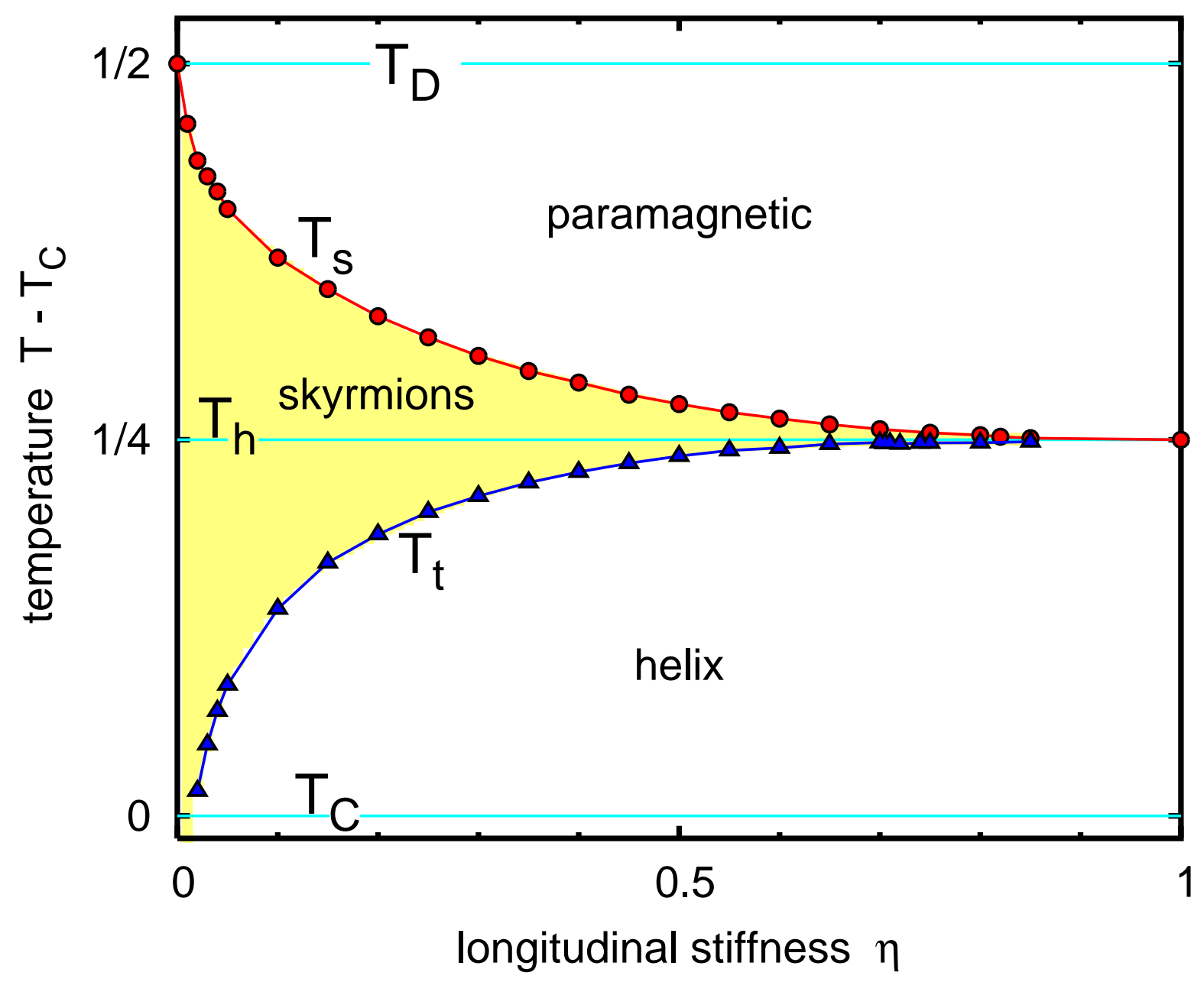

Fig. 2 
FIG. 2: Phase diagram of a chiral ferromagnet in terms of temperature versus longitudinal stiffnessparameter $(\eta, T)$. The diagram is based on the stability of the cylindrical skyrmions shown in Fig. 1c. Therefore, the diagram applies for two- and three-dimensions. Above the ferromagnetic Curie temperature $T_{C}$ two characteristic temperatures, defined in Eqn. (3), rule the phase diagram: $T_{D}$ for the instability against double twisting and $T_{h}$ for the formation of the helical phase. The skyrmion phase is thermodynamically stable between the line $T_{S}(\eta)$ for the continuous transition into the paramagnetic phase and the line $T_{t}(\eta)$ for the first-order transition into the helix. This line is independent on the chosen parameter $b$. The helical phase exists as a metastable phase between $T_{h}$ and $T_{t}(\eta)$. The estimate for the first-order line $T_{t}(\eta)$ has been calculated for models with $b=0.05$. 

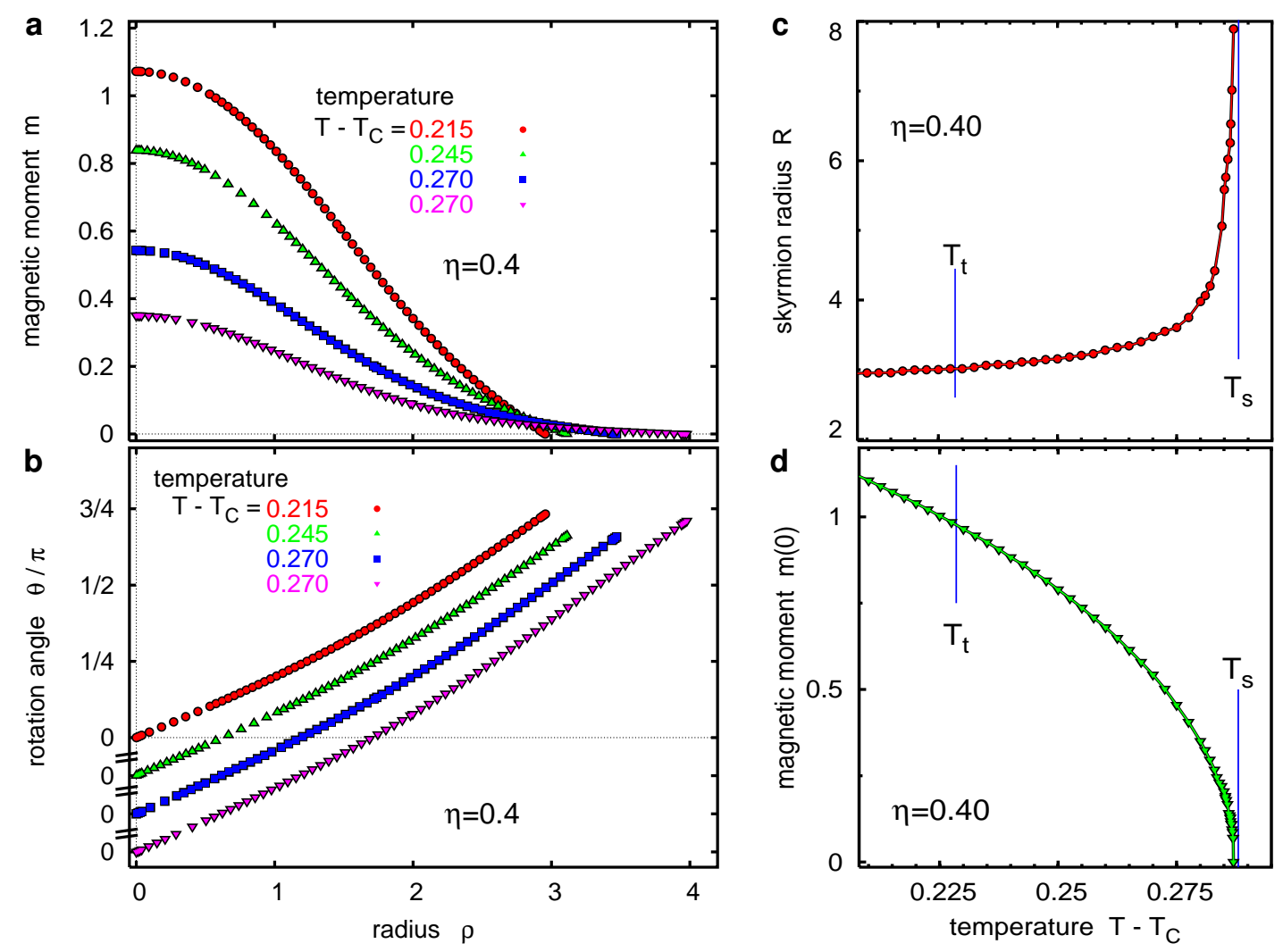

Fig. 3 
FIG. 3: Details of skyrmions solutions for $\eta=0.4$ and $b=0.05$ at various temperatures. (See, Eqs. (7), (8) in Methods section.) a, Variation of the modulus of the magnetisation as function of radius at different temperatures. $\mathbf{b}$, Variation of the angle of rotation, $\theta$, as function of radius at different temperatures. At low temperatures the magnetization rotates from angle zero in the center to angles $\theta(R)<\pi$ at the cell boundaries. The angles $\theta(R)$ depend on temperature. The radius is given in units of the twisting lengths $1 / q_{0}$. c , Evolution of the radius of the skyrmion cell up to the transition into the paramagnetic state. In the transition region both the radius of the skyrmion cell $R$ and the angles $\theta(R)$ grow unlimitedly by approaching the critical temperature $T_{S}$. d, Corresponding evolution for the amplitude magnetization in the center $m(0)$. The modulus gradually decreases to zero as the temperature approaches the critical value $T_{S}$ while the radius of the skyrmions diverges. By this process the single skyrmions forming a dense stable condensate below $T_{S}$ can transform into isolated skyrmions. Note that the characteristic twisting length in radial direction, which is given by the slope $\theta(\rho)$ in panel $\mathbf{b}$, does not change with changing temperature. The core region of the skyrmions with sizeable magnetization has essentially a fixed diameter, while only a tail with diverging radius and rapidly vanishing local magnetization evolves near $T_{S}$. This diameter is given by the ratio $D / A$, which also fixes the characteristic size of chiral fluctuations in the paramagnetic phase above $T_{S}$. 

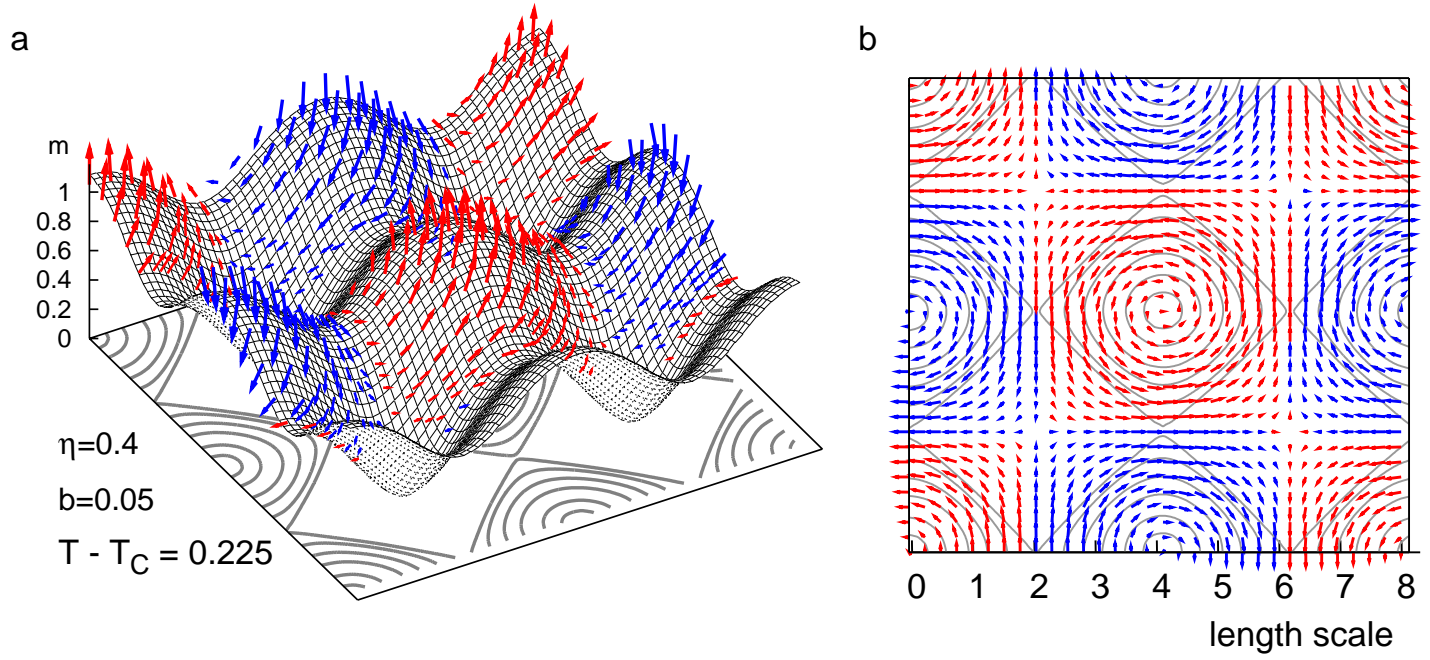

Fig. 4 
FIG. 4: Structure of a two-dimensional skyrmion lattice, derived as minimum energy solution for the model Eqn. (11) with Dzyaloshinskii-Moriya interactions, Eqn. (4). The ground-state has fourfold symmetric lattice structure, and net magnetization direction of the skyrmion cores is staggered. Thus, the periodicity of this square lattice is doubled as a $2 \times 2$ antiferromagnetic structure. a, overview showing the modulus $m$ and the corresponding magnetization vectors. $\mathbf{b}$, Projection of the magnetization vectors into the base-plane. Units shown in $\mathbf{b}$ are the twisting length $1 / q_{0}$. Red/blue signals local magnetization direction with positive/negative components arbitrarily taken perpendicular to the two-dimensional plane. For the core-regions of the skyrmions, lines of constant modulus, $m=$ const, are shown as contour lines in the base-plane. This result applies to thin magnetic films with broken inversion symmetry made from isotropic or cubic (crystallographic class T) metallic materials. The magnetization direction rotates around the radial directions going outward from the skyrmion center. A three-dimensional analogue of this dense texture composed of an amorphous arrangement of cylindrical skyrmion strings is consistent with recent experiments in the cubic B20 metals FeGe and MnSi [30, 31, 34] as discussed in the text. 